\title{
高速超声椭圆振动铣削腹板表面质量研究
}

\author{
高 泽 1,2 张德远 ${ }^{1,2}$ 李 哲 ${ }^{1,2}$ 姜兴刚 ${ }^{1,2}$ 刘佳佳 ${ }^{1,2}$ \\ (1. 北京航空航天大学机械工程及自动化学院 北京 100191; \\ 2. 北京航空航天大学先进加工技术研究中心 北京 100191)
}

\begin{abstract}
摘要: 针对航空航天常用的钛合金机械加工特性差、加工质量低、效率差的问题, 提出一种新的加工工艺, 高速超声粗圆振 动铁削工艺。它是一种刀尖在平面内按粗圆轨迹运动的超声频间歇性切削方法, 能够有效地改善铣削质量且突破了常规超声 振动加工对临界速度的限制。将高速超声振动铣削工艺引入航空常见薄壁零件结构, 腹板的加工中。先分析该工艺微观分离 原理，并设计试验验证该工艺在钛合金腹板加工中的效果。结果显示：高速超声振动铣削工艺相比于普通铁削的加工方式在 已加工表面让刀量及表面粗粘度值两方面均有稳定提升。粗粘度降幅约 10\% 40\%, 让刀量降幅 50\%左右。
\end{abstract}

关键词: 高速超声铣削; 粗圆振动; 钛合金; 薄壁腹板; 表面质量

中图分类号: V261

\section{Research on Surface Quality of Titanium Alloy Webs via High-speed Ultrasonic Elliptical Vibration Milling}

\author{
GAO Ze $\mathrm{Z}^{1,2}$ ZHANG Deyuan ${ }^{1,2}$ LI Zhe ZHANG Xinggang $^{1,2}$ LIU Jiajia ${ }^{1,2}$ \\ (1. School of Mechanical Engineering and Automation, Beihang University, Beijing 100191; \\ 2. Research Center for Advanced Processing Technology, Beihang University, Beijing 100191)
}

\begin{abstract}
To solve the problems of the poor machining performance, low processing quality and poor efficiency of titanium alloys commonly used in aerospace, a new processing technology, high-speed ultrasonic elliptical vibration milling process is proposed. It is an ultrasonic intermittent cutting method in which the tip of the blade moves in an elliptical path in the plane, which can effectively improve the quality of milling and break through the limitations of the conventional ultrasonic vibration processing on the critical speed. High-speed ultrasonic vibration milling process is introduced into the common thin-walled parts structure, web processing. The principle of microscopic separation of the process is analyzed first, and the experiment is designed to verify its effect in the processing of titanium alloy webs. The results show that the high-speed ultrasonic vibration milling process has a stable increase in both the number of cutters and the surface roughness value in the processed surface compared to the ordinary milling processing method. The roughness reduction is about $10 \%$ to $40 \%$, and the relieving amount reduction is about $50 \%$.
\end{abstract}

Key words: high-speed ultrasonic milling; elliptical vibration; titanium alloy; thin-wall web; surface quality

\section{0 前言}

钛合金密度较低, 同时强度较高, 这使得与其 它金属相比钛合金比强度、比刚度更高 ${ }^{[1]}$, 更适合 做关键的承重部件。而对于飞行器的结构设计来说, 在保证其强度、刚度的条件下, 减轻各部分结构的 重量、提升各部分构件之间的整体性, 是发展的必 然趋势 ${ }^{[2]}$ 。钛合金的优良特性已让它广泛地被接受 与使用 ${ }^{[3]}$, 钛合金正成为航空工业最重要的工程材

20180411 收到初稿, 20180920 收到修改稿
料之一[4]。

在壳体件、平板件中, 当零件壁厚与内径曲率 半径或轮廓尺寸之比小于 1:20 的时候, 将称之为薄 壁零件。腹板作为一种常见的薄壁结构, 自身刚度 低加工过程中受力易变形, 加工余量大。目前薄壁 零件加工变形控制的优化研究集中在改良刀具的结 构和改良走刀路径、切削参数、刀具参数等 ${ }^{[5-6]}$, 或 是适当分配加工余量、在线数控补偿技术等辅助支 撑 ${ }^{[7-8]}$ 。同时加工过程中易颤振, 加工表面质量差。 目前研究局限于颤振的预报和模型建立阶段。薄壁 加工的稳定性与加工变形都会严重影响零件的性能 指标, 进而影响其正常工作。 
传统超声加工技术自提出至今已有七十余年的 历史 ${ }^{[9]}$, 超声加工已被引入到了车削, 铣削等多个 领域, 对加工效果有明显改善 ${ }^{[10]}$ 。超声振动模式有 轴向、弯曲、扭转三种基本形式以及它之间复合等 多种形式, 目前以轴向振动(纵振)以及双向弯曲振 动(椭圆振动)研究应用最为广泛。超声振动的引入 将传统连续铣削过程变成断续的铣削过程, 对以下 几方面的提升有着明显效果 ${ }^{[11-21]}$ : KIM 提出在金刚 石加工中超声辅助可降低切削力的观点 ${ }^{[11]}$; ZHANG 等 ${ }^{[13]}$ 提出超声铣削可降低脆性材料加工中 的切削力; ABDULLAH ${ }^{[14]}$ 提出不同振幅下, 超声 振动降低切削力效果也不同。SHEN 等 ${ }^{[12]}$ 提出超声 辅助可明显改善槽侧壁铣削加工的粗糙度; 高国富 等 ${ }^{[15-17]}$ 分别提出了在碳纤维复材、陶瓷、铝材加工 中超声辅助可以降低表面粗粘度、CHERN 等 ${ }^{[19]}$ 提 出振动辅助加工还可以改善槽加工的尺寸精度; RAZFAR 等 ${ }^{[20]}$ 提出超声辅助铣削钢材时精度得到 提升; SHEN 等 ${ }^{[18]}$ 提出超声振动有利于铣削加工切 屑变小; 这些优良效果保证的超声加工的广泛应 用 ${ }^{[21]}$ 。为了保证超声加工效果, 超声振动轨迹需要 分离, 加工速度存在一个临界值, 影响加工效率。

本文将高速超声理念 ${ }^{[22]}$ 引入铁削加工过程中, 以进一步提升铣削加工质量、效率。并设计试验验 证其在薄壁腹板加工中的效果。首先分析该工艺分 离轨迹原理, 然后通过试验将其与普通铣削加工对 比, 在一定的工艺参数范围内, 高速超声振动铣削 工艺都能够有效地降低已加工表面的粗粘度和让刀 量最后结合得到的数据进行分析讨论。说明了该工 艺在突破了临界速度时也能产生有效分离, 在薄壁 零件加工上有提升表面质量的优良特性。

\section{1 高速超声椭圆振动铣削原理分析}

高速超声椭圆振动铣削是在普通铣削基础上, 给刀具整体附加一个椭圆振动。以刀具回转轴为 $Z$ 轴, $X 、 Y$ 所在平面垂直于 $Z$ 轴且三坐标轴相互垂直 建立直角坐标系。以图 1 中坐标系为基准建立刀尖 轨迹方程。工件即为试验用工件示意图, 工件中部 横梁长度 $L$ 远大于其厚度 $b$ 。普通铣削过程刀尖上 的一点 $N$ 轨迹是由主轴的回转运动和进给运动两部 分合成, 方程为

$$
\left\{\begin{array}{l}
x=x_{r}+x_{v}=\frac{D}{2} \cos \omega_{r} t+\frac{v t}{60} \\
y=y_{r}+y_{v}=\frac{D}{2} \sin \omega_{r} t \\
z=0
\end{array}\right.
$$

式中, $D$ 为铣刀直径; $\omega_{r}$ 为主轴回转角速度; $v$ 为 进给速度。

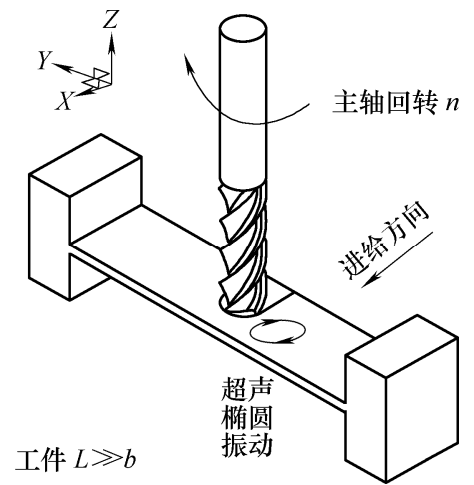

图 1 试验用工件坐标轴

当刀尖振动的椭圆轨迹长短轴与设定的 $X 、 Y$ 轴重合时，椭圆振动轨迹方程为

$$
\left\{\begin{array}{l}
x_{t}=A \cos \omega_{f} t \\
y_{t}=B \sin \omega_{f} t \\
z_{t}=0
\end{array}\right.
$$

式中, $A 、 B$ 为长短轴方向超声振动振幅; $\omega_{f}=2 \pi f$; $f$ 为超声激振频率。

而当铣刀转过 $\alpha$ 角之后, $N$ 点在 $X 、 Y$ 坐标系 中轨迹方程需要做一个变换

$$
\left\{\begin{array}{l}
x=A \cos \omega_{f} t \cos \alpha-B \sin \omega_{f} t \sin \alpha \\
y=A \cos \omega_{f} t \sin \alpha+B \sin \omega_{f} t \cos \alpha
\end{array}\right.
$$

式中, $\alpha=\omega_{r} t$ 为 $t$ 时刻转过的角度。

将上面式子叠加得到任意时刻刀尖 $N$ 点轨迹 方程

$\left\{\begin{array}{l}x=\frac{D}{2} \cos \omega_{r}+\frac{v t}{60}+A \cos \omega_{f} t \cos \alpha-B \sin \omega_{f} t \sin \alpha \\ y=\frac{D}{2} \sin \omega_{r}+A \cos \omega_{f} t \sin \alpha+B \sin \omega_{f} t \cos \alpha \\ z=0\end{array}\right.$

一般的, 第 $m$ 个刀尖上点 $N_{\mathrm{m}}$ 轨迹方程为

$$
\left\{\begin{aligned}
x= & \frac{D}{2} \cos \omega_{r}\left(t-\frac{(m-1) \pi}{2}\right)+\frac{v t}{60}+A \cos \omega_{f} t \cos \alpha- \\
& B \sin \omega_{f} t \sin \alpha \\
y= & \frac{D}{2} \sin \omega_{r}\left(t-\frac{(m-1) \pi}{2}\right)+A \cos \omega_{f} t \sin \alpha+ \\
& B \sin \omega_{f} t \cos \alpha
\end{aligned}\right.
$$

由该公式可以画出刀尖运动轨迹图, 如图 2 所 示。式中未知参数根据试验参数 $D=12 \mathrm{~mm}$, $\omega_{r}=2 \pi \times 2660$ (刀尖线速度 $v=100 \mathrm{~m} / \mathrm{s}$ ),$v=106.32$ $\mathrm{mm} / \mathrm{min}$ (每齿进给量 $f_{z}=0.01 \mathrm{~mm}$ ), $A=B=11 \mu \mathrm{m}$, $\omega_{f}=2 \pi \times 17880$ 。刀尖轨迹如下图。其中 $v>2 \pi A f$ 切 
削速度已超过临界速度, 每一齿自身加工过程中并 无分离, 高速超声振动加工是通过控制每齿之间相 位关系, 凭借相邻两齿之间轨迹叠加来制造分离效 果, 如下图所示, 经过第一齿切削, 第二齿在 $A_{1} A_{2}$ 这一个切削周期中只有 $B D$ 段参与加工, $A_{1} B$ 和 $D A_{2}$ 两阶段都是空切。

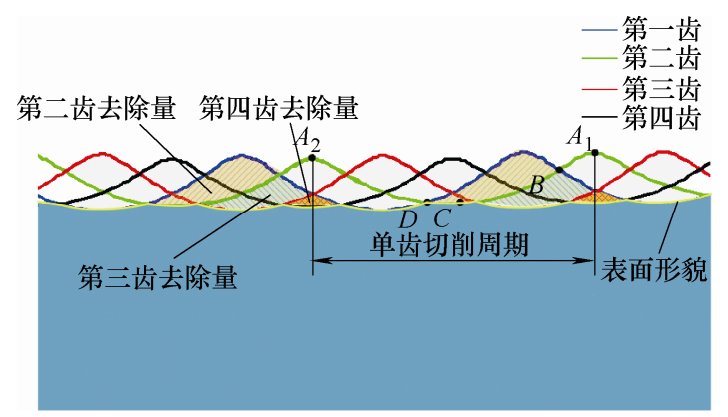

图 2 刀尖轨迹分离情况

从图 2 可知确保高速超声铣削工艺分离的关键 是控制相邻齿之间相位关系。对于 $m$ 齿铣刀, 当主 轴转数为 $n$ 时, 后一齿落后前一齿时间为 $\frac{60}{m n} \mathrm{~s}$, 超 声振动频率为 $f$, 则后一齿落后前一齿振动周期数

$$
\omega_{1}=\frac{60 f}{m n}
$$

产生每齿进给量 $f_{z}$ 大小的振动需要时长为 $\frac{f_{z}}{v}$, 则由进给造成的振动周期数差值为

$$
\omega_{2}=\frac{f_{z}}{v} \times f=\frac{60 f f_{z}}{n \pi d}
$$

相邻两齿相差的振动周期数自然是: $\omega_{3}=\frac{1}{m}$ 。

如图 2 易知只要相邻两齿相差的周期数 $\omega=\omega_{1}+\omega_{2}+\omega_{3} \neq 1$ 即可, 常数 $\omega \in(0,1)$, 都可以在 加工过程中产生分离, 本试验中取 $\omega=0.25$ 。

同时, 在刀具转过任意 $\alpha$ 角后, 刀尖 $\mathrm{N}$ 点法向切 削厚度 $h=f_{z} \cos \alpha<A+B$, 本试验中 $A+B=22 \mu \mathrm{m}$, $f_{z}<A+B$ 可保证刀具旋转一周中都有分离。椭圆 振动铣削是指刀尖在平面内按椭圆轨迹运动, 本试 验使用装置两个相互垂直方向振动独立产生, 并合 成平面内振动, 只是长短轴振幅刚好相同, 原理仍 符合椭圆振动, 振动的发生与分离情况也都和椭圆 振动相同。可认为轨迹为特殊的椭圆。

\section{2 试验}

\section{1 试验平台设计}

为验证高速超声振动铣削在腹板加工中的效 果, 设计了图 3 所示试验系统。该系统主要由加工
中心、工件、超声振动系统三部分组成。试验用加 工中心为 BV100 立式加工中心。试验用工件为图 1 中的工字型钛合金(Ti-6Al-4V)腹板零件, 厚度 3 $\mathrm{mm}$, 宽 $42 \mathrm{~mm}$, 长(即左右翼板距离) $100 \mathrm{~mm}$ 。工 件固定在测力仪上方, 试验过程中可以直接采集力 数据, 测力仪固定于加工中心工作台。超声振动系 统由超声电源、供电装置、铣削换能器组成, 超声 电源产生频率为 $17880 \mathrm{~Hz}$ 激励, 经供电装置传输, 使换能器产生稳定的超声频振动, 换能器变幅杆末 端是直径为 $12 \mathrm{~mm}$ 的标准四刀硬质合金铣刀，在激 励下刀具尖端产生稳定的 $X 、 Y$ 方向振幅皆为 $11 \mu \mathrm{m}$ 椭圆振动。

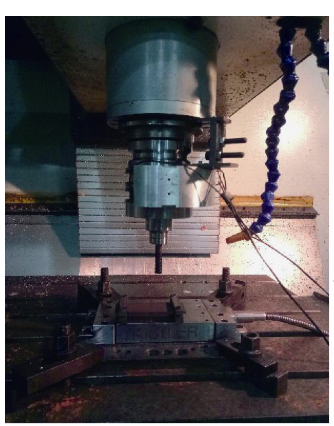

(a) 实物平台

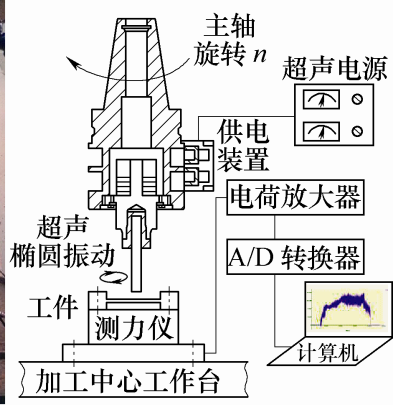

(b) 原理示意图
图 3 试验平台

\section{2 试验方案设计}

试验方案设计如下: 铣刀加工位置为腹板正中 心, 从一端走刀至另外一端, 为避免加工过程中影 响因素干扰, 同参数超声与普通加工在一次走刀中 完成, 通过控制超声电源开关转换超声、普通加工 模式, 试验中先进行超声加工, 再进行普通加工。

目前工厂常用钛合金铣削切削速度为 $80 \mathrm{~m} / \mathrm{s}$ 。 本试验为突出高速超声铣削工艺在效率上的优势 使用更高切削速度, 每齿进给量则根据刀尖振动幅 值合适变化, 铣削厚度取固定值。具体参数如表 1 所示。

表 1 加工参数表

\begin{tabular}{cc}
\hline 参数 & 数值 \\
\hline 切削速度 $/(\mathrm{m} / \mathrm{s})$ & $80 、 100 、 120 、 140 、 160$ \\
每齿进给量 $/ \mathrm{mm}$ & $0.01 、 0.015 、 0.02$ \\
切深 $/ \mathrm{mm}$ & 0.2 \\
\hline
\end{tabular}

其中切削速度 $v=n \pi D, D$ 为试验用铣刀直径, $n$ 为机床主轴转速, 即切削速度与主轴转速成正比 例, 试验参数中切削速度的变化即机床转速的变化。

\section{3 试验结果测量}

本试验主要观测加工后表面粗粘度值, 让刀量 两方面结果, 粗䊁度值使用 zygo 白光干涉仪扫描轮 廓并测量表面粗粘度值, 测量精度为 $0.001 \mu \mathrm{m}$, 在 
扫描视野中随机取三条线上的粗糙度值, 取平均, 作为试验最后测量结果。为完整表征表面形貌，还 使用相机拍摄已加工表面宏观外形轮廓, 并使用扫 描电镜, 型号 JSM 6010 分别拍摄超声加工与普通 加工放大 100 倍之后的表面形貌作对比。让刀量通 过螺旋测微仪测量加工后表面剩余厚度来衡量, 螺 旋测微仪型号为日本三丰量具 mitutoyo, 测量区域 为直径 $4 \mathrm{~mm}$ 的圆形, 测量精度为 $0.001 \mathrm{~mm}$, 测量 区域为超声加工入刀位置中心和普通加工出刀位置 中心, 两位置关于整个薄板对称, 以便减少其他因 素干扰。试验切削力通过 Kistler 9254 型测力仪进行 测量, 测力仪采样频率 $50000 \mathrm{~Hz}$, 可测量 $X 、 Y 、 Z$ 及其复合的多方向力数据, 测量范围为 $0 \sim 20 \mathrm{kN}$, 精度为 $0.01 \mathrm{~N}$ 。

\section{3 试验结果与讨论}

\section{1 粗糙度分析}

在切深 $0.2 \mathrm{~mm}$, 每齿进给量分别为 $0.01 \mathrm{~mm}$ 、 $0.015 \mathrm{~mm} 、 0.02 \mathrm{~mm}$ 时, 已加工表面粗糙度值随切 削速度变化如图 4 所示。

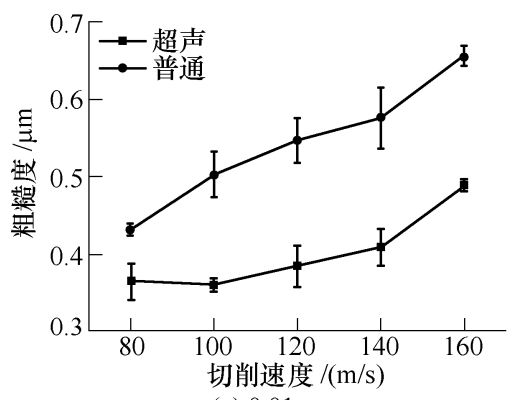

(a) $0.01 \mathrm{~mm}$

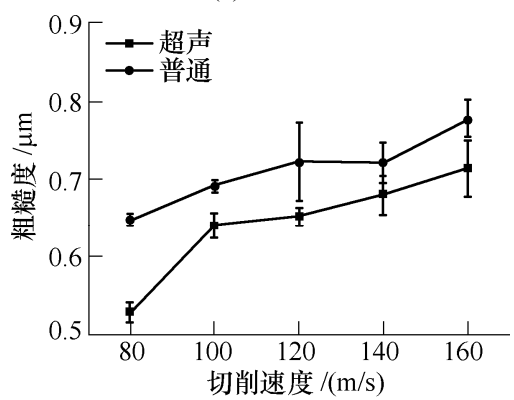

(b) $0.015 \mathrm{~mm}$

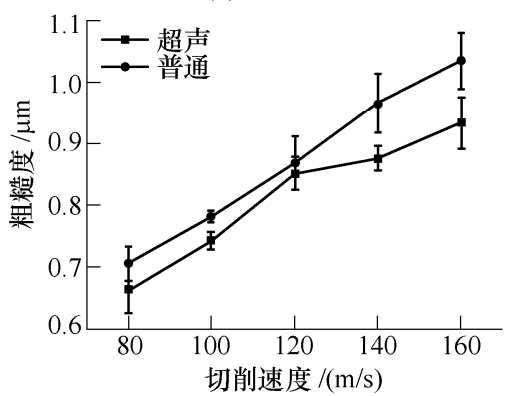

(c) $0.02 \mathrm{~mm}$

图 4 不同每齿进给量下粗粘度随切削速度变化
从图 4 中可以看出高速超声铣削工艺在试验用 参数下都能够稳定地实现粗粘度值下降。通过超声 工艺带来的下降率可以发现, 在固定每齿进给量不 变时, 变化切削速度, 下降率变化规律不明显, 可 认为切削速度对下降率无明显影响。而固定切削速 度值, 改变每齿进给量, 随着每齿进给量增大, 降 低率也在下降, 每齿进给量 $f_{z}=0.01 \mathrm{~mm}$ 时下降率 最明显, 在 $25 \%$ 左右, $f_{z}=0.015 \mathrm{~mm}$ 时下降率仅有 $10 \%$ 左右, $f_{z}=0.02 \mathrm{~mm}$ 时下降率又略有降低, 而 每齿进给量直接影响超声工艺分离情况，有效的 分离可以突出超声工艺对于表面粗糙度值降低的 效果。为了在实际加工中得到更好的表面粗䊁度 值，应增大超声振动幅值或者适当降低每齿进给 量值。若同时希望保持较大的总进给量, 由总进 给量 $F=m f_{z} \times n$, 即铣刀齿数乘每齿进给量乘以 机床主轴转速, 可以选择适当增大切削速度, 切 削速度增大对超声工艺降低粗粘度值比率没有明 显影响。

使用相机拍摄试验工件宏观表面形貌，如图 5 所示。在完全相同的试验参数、条件下，高速超声 铣削工艺加工出来的宏观表面有着较为明显的差 别。从图中可见普通加工出的表面相比于超声加工 出的表面更亮。图 5 中左下对应普通铣削局部放大, 右下对应超声铣削局部放大。局部放大后可以观察 到普通加工出的表面每一齿切过的痕迹清晰明显, 整个表面由多个单齿加工形成的圆弧组成，而超声 加工过的表面, 每齿加工过程中都形成了均匀致密 的更为微细的结构。

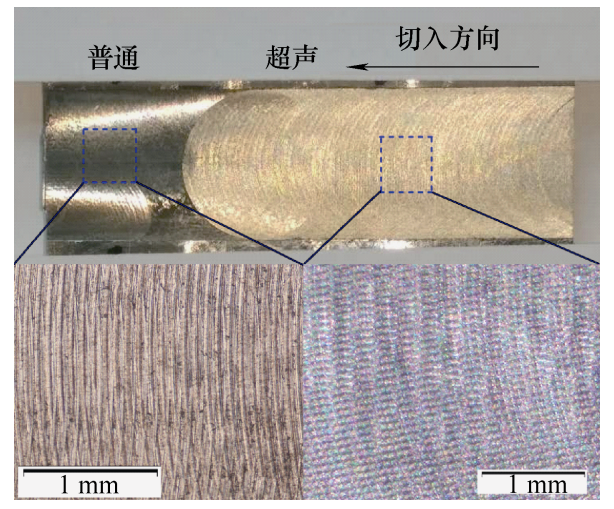

图 5 试验工件宏观表面形貌

采用电子显微镜拍摄的已加工表面微观形 貌图，结果如图 6 所示。从图中可见放大倍数为 100 倍时, 能够明显地看到普通加工得到的表面 只是由每齿加工形成的圆弧构成, 而超声加工 过的表面，则留有超声频冲击产生的均一致密 的纹理。 


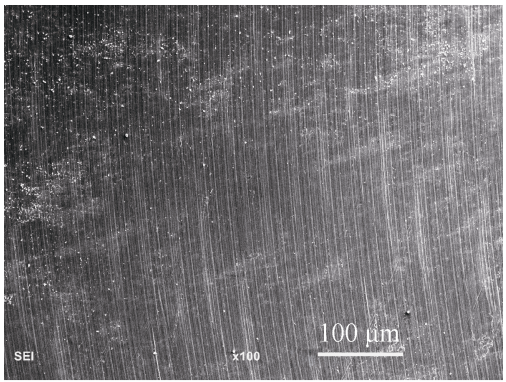

(a) 普通加工

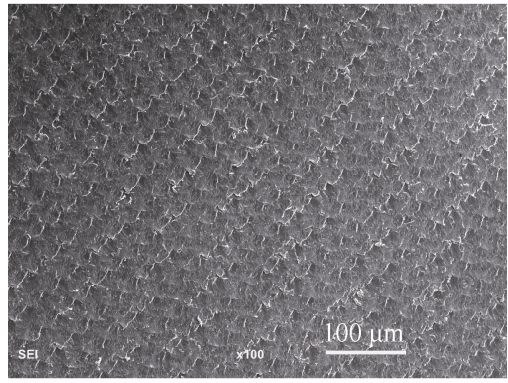

(b) 超声加工

图 6 普通与超声加工试验件电子显微镜下形貌

使用白光轮廓扫描仪得到的超声与普通加工表 面微观形貌, 如图 7 所示, 从图中可以发现超声加 工得到的表面明显更为平整, 微小的峰谷间距离也 更小, 超声带来的间歇加工特性有利于减少加工中 的振动，得到更平整、光滑的表面。

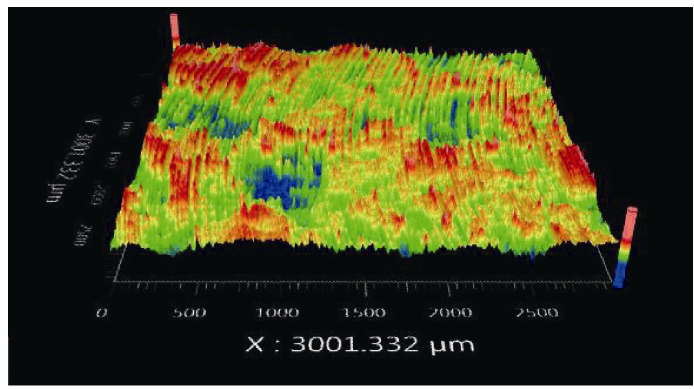

(a) 普通加工

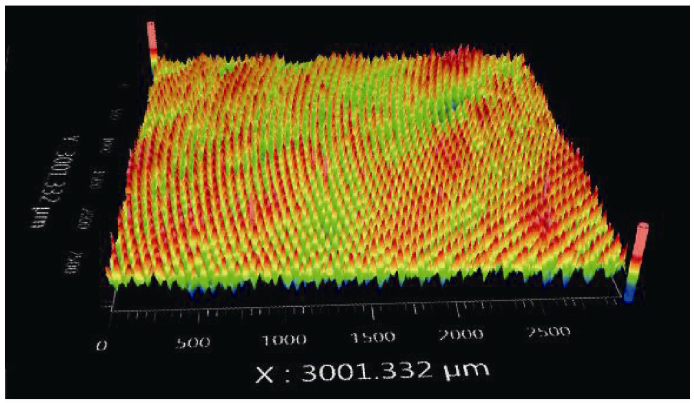

(b) 超声加工

图 7 普通与超声加工表面白光扫描形貌图

结合已加工表面宏观至微观形貌图片可以发 现: 超声振动加工的分离特性, 使得每次刀尖加工 切削过程去除量更小, 有效避免了加工过程中较大 的振动, 使得得到的表面更均匀平整没有明显的缺 陷。稳定的超声振动使得每齿加工后的表面峰谷分
布更为均匀细密, 峰谷差值稳定在一定程度之内。 这些原因使得高速超声工艺下得到的表面粗鋉度值 有一定程度降低。

\section{2 让刀量分析}

加工过程中，切削力是工件与刀具相互作用的 结果，也是薄壁产生让刀，导致加工精度下降的主 要因素。图 8 是切深 $0.2 \mathrm{~mm}$, 切削速度 $120 \mathrm{~m} / \mathrm{s}$, 每齿进给量 $0.02 \mathrm{~mm}$ 试验参数下测力仪测得的切削 力变化曲线。
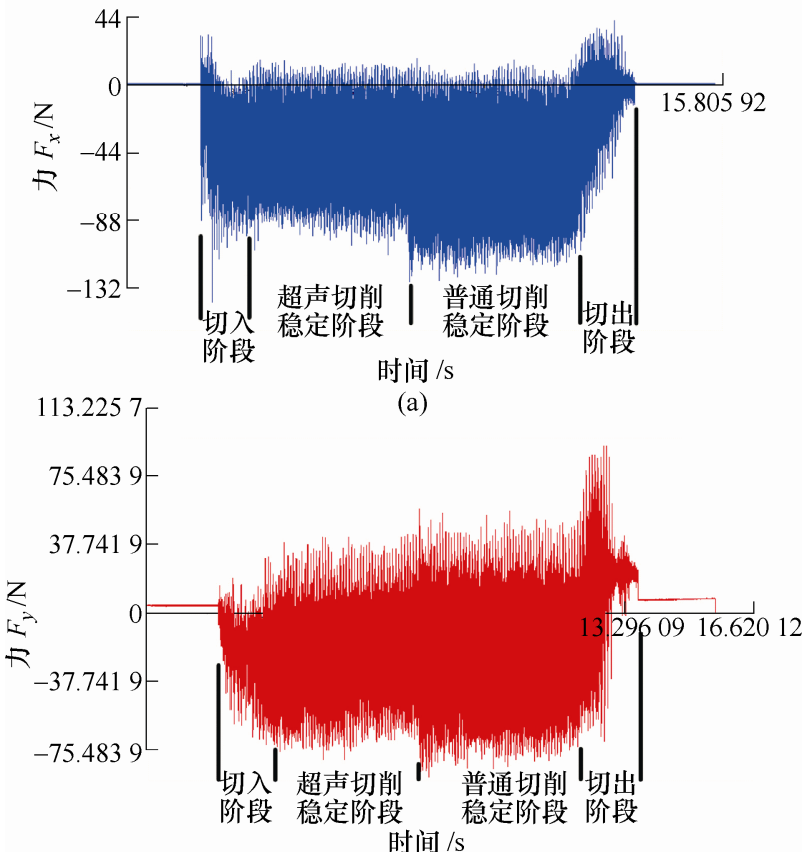

(b)

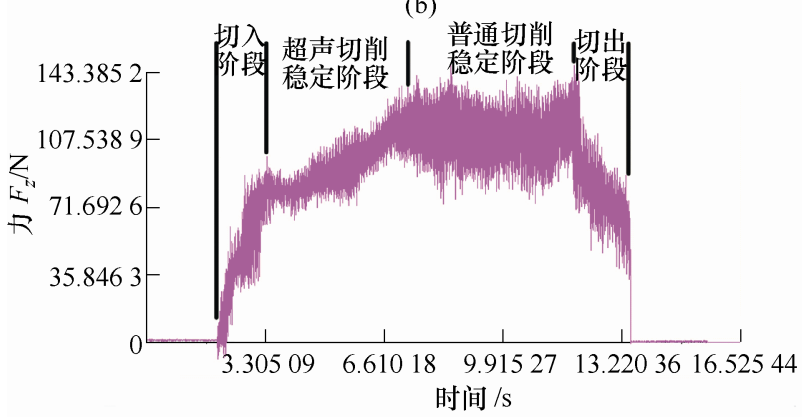

(c)

图 $8 X 、 Y 、 Z$ 方向力实测曲线图

图中 $F_{x}, F_{y}$ 方向的力大部分处于负半轴, 表示 测力仪测得的力数据值与测力仪默认正方向相反， 故在讨论力数值大小时关注其绝对值即可。从图 8 可见, 试验中腹板加工过程中力变化主要分为以下 几个阶段: 切入阶段、超声切削稳定阶段、普通切 削稳定阶段、切出阶段。切入阶段铣刀端面逐渐接 触工件, 三个方向力均从零增长至某一值, 铣刀端面 完全进给至腹板之上后力开始稳定，为超声切削稳定 阶段, 在加工进行一半左右, 手动关闭电源, 此时开 始普通切削阶段，力的数值发生一定改变，随后刀具 
继续沿进给方向运动, 逐渐与工件分离, 此时为切出 阶段, 力在冲击下产生一定波动随后降到零。

本试验主要关注稳定切削时超声铣削与普通铣 削切削力大小, 故在图中切削稳定阶段, 使用软件的 enable mean value 功能直接得到该区域中平均力的大 小。图 9 为切深 $0.2 \mathrm{~mm}$, 每齿进给量 $0.02 \mathrm{~mm}$ 时 $X$ 、 $Y 、 Z$ 三个方向切削力随切削速度变化的对比图。
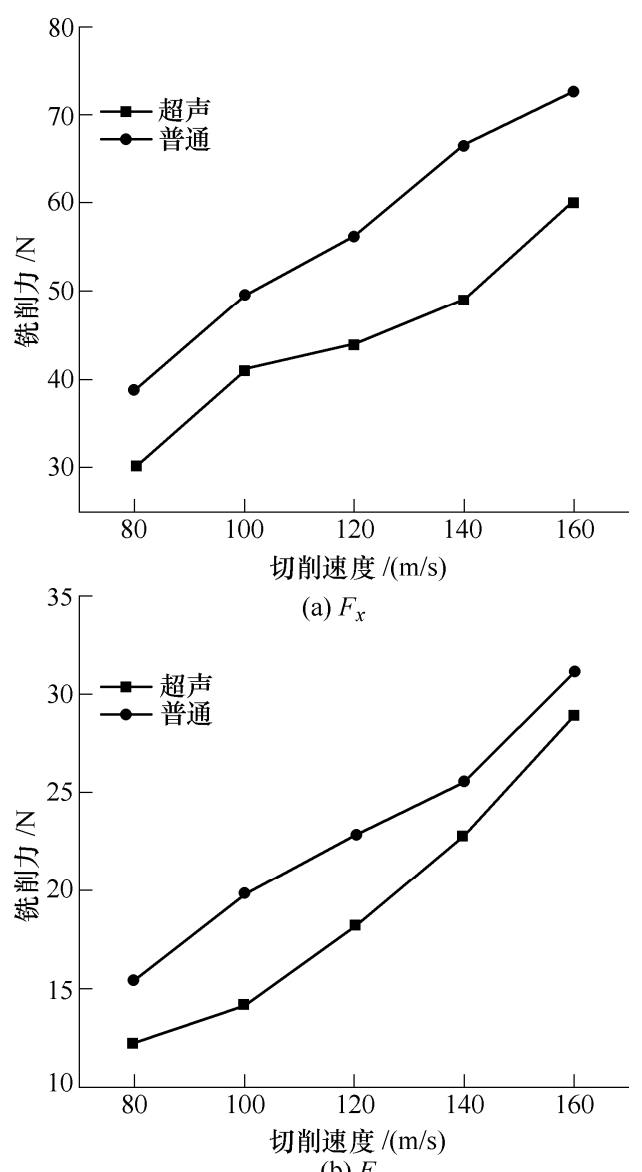

(b) $F_{y}$

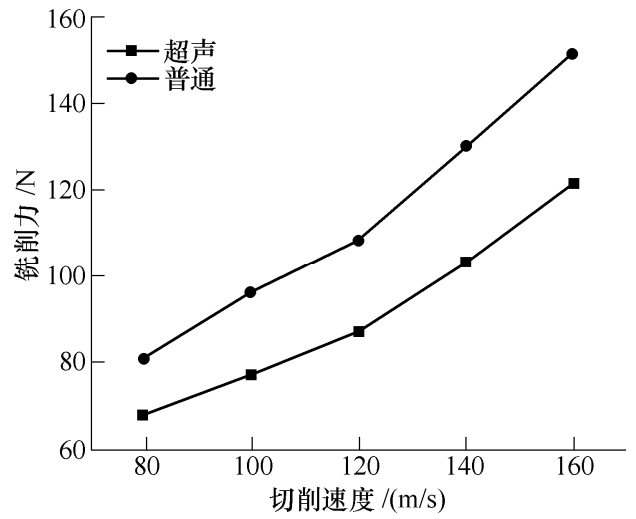

(c) $F_{z}$

图 9 每齿进给量为 $0.02 \mathrm{~mm}$ 时切削力随切削速度变化

从图 9 中我们可以发现高速超声铣削工艺对腹 板加工过程中工件受到的 $X 、 Y 、 Z$ 三个方向切削力 都有明显的降低作用, 其中 $X$ 方向为铣刀进给方向, 在此参数下 $X$ 方向力降低率为 $21 \%$ 。 $Y$ 方向与 $X$ 在
同一平面内且相互垂直, 受到的力最小, 力下降幅 度在三个方向中最不明显, 约 $17 \%$ 。 $Z$ 方向为铣刀 轴线方向, 是腹板加工过程中主要受力方向, 受到 的力最大，超声工艺约使力下降 19\%。

在切深 $0.2 \mathrm{~mm}$, 每齿进给量分别为 $0.01 \mathrm{~mm}$ 、 $0.015 \mathrm{~mm} 、 0.02 \mathrm{~mm}$ 时, 已加工表面让刀量随切削 速度变化如图 10 所示。
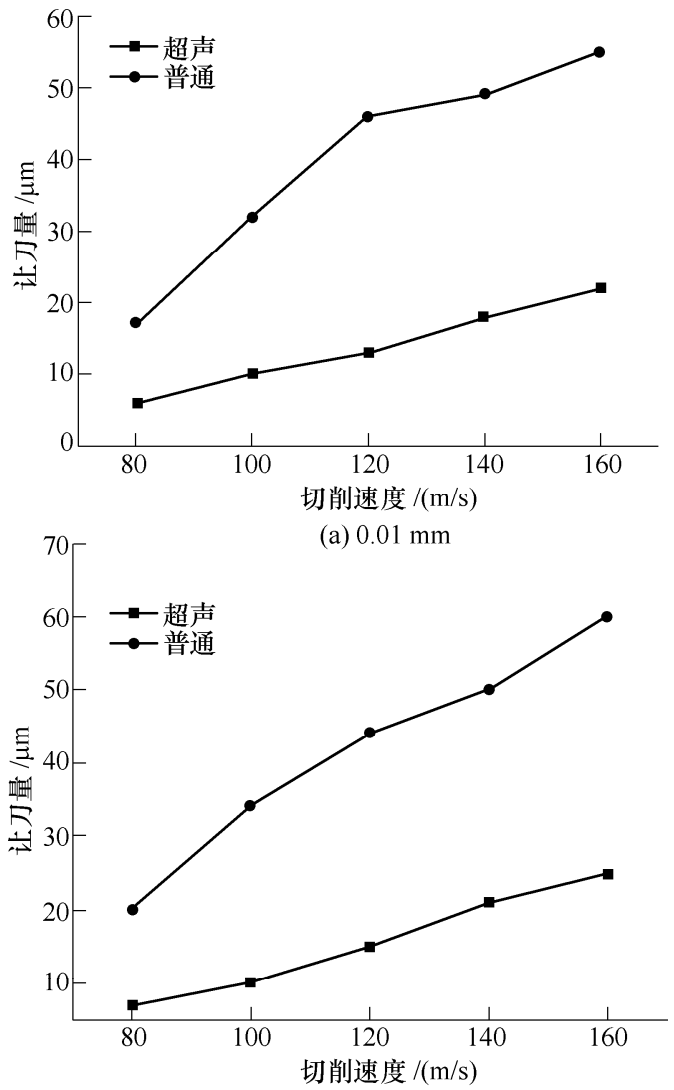

(b) $0.015 \mathrm{~mm}$

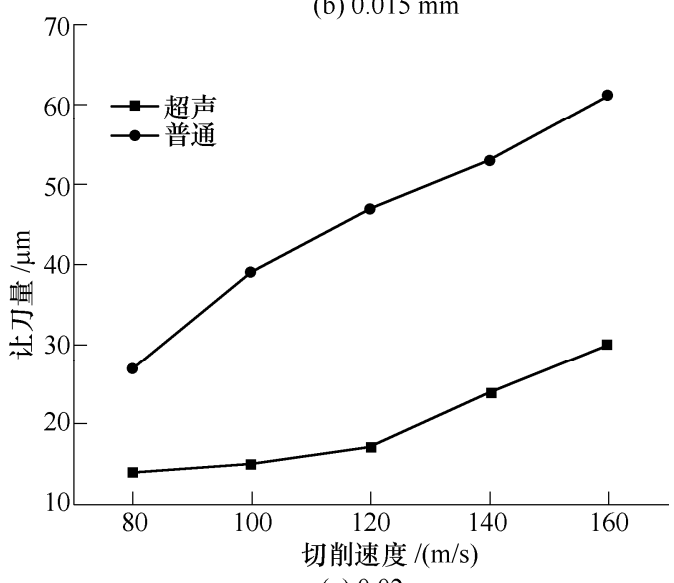

(c) $0.02 \mathrm{~mm}$

图 10 不同每齿进给量下让刀量随切削速度变化

从图 10 中可以发现高速超声铣削工艺在试验 用参数下都能够稳定实现让刀量减小，且降低率都 很明显。在每齿进给量不变时, 改变切削速度让刀 量降低率变化不明显。而控制切削速度不变, 增大 每齿进给量时, 降低率略有下降。 $f_{z}=0.01 \mathrm{~mm}$ 时, 
五组切削速度平均下降率为 $65.7 \%, f_{z}=0.015 \mathrm{~mm}$ 时, 五组切削速度平均下降率为 $63.56 \%, f_{z}=0.02$ $\mathrm{mm}$ 时, 五组切削速度平均下降率为 $55.46 \%$, 整体 略有下滑。

高速超声椭圆振动铣削分离特性使得一个刀齿 连续加工过程中的切削力变为脉冲力且切削过程中 会产生刀具切削角度的动态变化, 在原本刀尖的一 个连续加工周期部分变为空切过程, 去除厚度也变 小。这些因素都会降低铣削力。在薄壁件加工中, 让刀量主要受切削力影响, 超声的分离机制带来的 断续切削可以有效降低加工过程中的平均力, 进而 明显降低让刀量。

\section{4 结论}

(1) 高速超声椭圆振动铣削加工工艺突破传统 超声铣削工艺对速度限制, 有效改善了腹板加工中 表面粗糙度值大, 表面质量差及让刀量大, 加工精 度差两方面，扩大了超声振动铣削的应用范围。

(2) 相比于普通铣削, 高速超声振动铣削能够 降低加工表面的表面粗䊁度值 10\% 40\%。加工得 到的表面也更为平整、光滑。

(3) 相比于普通铣削, 高速超声椭圆振动加工 过程中工件所受到的切削力更小，降幅约 $20 \%$, 同 时腹板让刀下降 50\%左右, 得到的工件精度更好, 有助于直接提升腹板类薄壁件的加工精度。

\section{参 考 文 献}

[1] LEYENS C, PETERS M. Titanium and titanium alloys [M]. Weinheim: Wiley-VCH, 2003.

[2] 王盛璋. 航空钛合金复杂结构件铣削刀具性能综合评 判技术研究[D]. 南京: 南京航空航天大学, 2015 .

WANG Shengzhang. Study on comprehensive evaluation for cutting tools in milling aviation Ti-alloy components[D]. Nanjing : Nanjing University of Aeronautics and Astronautics, 2015.

[3] PETERS M, KUMPFERT J, WARD C H, et al. Titanium alloys for aerospace applications[J]. Advanced Engineering Materials, 2003, 5(6): 419-427.

[4] LI A, ZHAO J, LUO H, et al. Progressive tool failure in high-speed dry milling of Ti-6Al-4V alloy with coated carbide tools[J]. International Journal of Advanced Manufacturing Technology, 2012, 58: 465-478.

[5] TLUSTY J, SMITH S, BADRAWY S, et al. Design of a high speed milling machine for aluminum aircraft parts $[\mathrm{J}]$. ASME Mfg Science and Eng., 1997, 2(0): 323-32.

[6] TLUSTY J, SMITH S, WINFOUGH W R. Techniques for the use of long slender end mills in high-speed milling[J]. CIRP Annals-Manufacturing Technology, 1996, 45(1): 393-6.

[7] 梅中义, 王运巧, 范玉青. 飞机结构件数控加工变形控 制研究与仿真[J]. 航空学报, 2005, 26(2): 234-239.

MEI Zhongyi , WANG Yunqiao, FAN Yuqing. Researching and simulating deformation of aerocraft structure part in NC machining[J]. Acta Aeronautica Et Astronautica Sinica， 2005, 26(2): 234-239.

[8] 王运巧, 梅中义, 范玉青. 航空薄壁结构件数控加工变 形控制研究 $[J]$. 现代制造工程，2005，1(1): 31-33.

WANG Yunqiao, MEI Zhongyi, FAN Yuqing. Research on deformation in NC machining of aerospace thin-walled structure part $[\mathrm{J}]$. Modern Manufacturing Engineering, 2005, 1(1): 31-33.

[9] 牛国玲, 赵黎杰, 顾立志, 等. 振动切削机理的研究状 况[J]. 佳木斯大学学报，2005，23(3): 479-482.

NIU Guoling, ZHAO Lijie, GU Lizhi, et al. The research state of the vibration cutting mechanism[J]. Journal of Jiamusi University, 2005, 23(3): 479-482.

[10] 张德远. 中国的超声加工[J]. 机械工程学报, 2017, 53(19): 1-2.

ZHANG Deyuan. Ultrasonic processing in China $[\mathrm{J}]$. Journal of Mechanical Engineering, 2017， 53(19): 1-2.

[11] KIM G D, LOH B G. An ultrasonic elliptical vibration cutting device for micro V-groove machining: Kinematical analysis and micro V-groove machining characteristics[J]. Journal of Materials Processing Technology, 2007 , 190(1-3): 181-188.

[12] SHEN X H, ZHANG J, XING D X, et al. A study of surface roughness variation in ultrasonic vibration-assisted milling[J]. International Journal of Advanced Manufacturing Technology, 2012, 58(5-8): 553-561.

[13] ZHANG C, ZHANG J, FENG P. Mathematical model for cutting force in rotary ultrasonic face milling of brittle materials[J]. International Journal of Advanced Manufacturing Technology, 2013, 69(1-4): 161-170.

[14] ABDULLAH A. Investigation of the effect of cutting speed and vibration amplitude on cutting forces in ultrasonic-assisted milling $[\mathrm{J}]$. Proceedings of the Institution of Mechanical Engineers Part B Journal of Engineering Manufacture, 2011，226(10): 1-7.

[15] 高国富, 马星辉, 董小磊. 超声振动铣削碳纤维复合材 料表面特征研究[J]. 现代制造工程，2009，2009(9): 86-89.

GAO Guofu, MS Xinghui, DONG Xiaolei. Research on the surface characteristics in ultrasonic milling of CFRP $[J]$. Modern Manufacturing Engineering, 2009, 29(9): 86-89. 
[16] GRAŽEVIČIŪTÉ J, SKIEDRAITĖ I, JŪRĖNAS V, et al. Applications of high frequency vibrations for surface milling[J]. Mechanika, 2015, 69(1): 46-49.

[17] SHEN X H, ZHANG J, XING D X, et al. A study of surface roughness variation in ultrasonic vibrationassisted milling[J]. International Journal of Advanced Manufacturing Technology, 2012, 58(5-8): 553-561.

[18] SHEN X H, ZHANG J H, LI H, et al. Ultrasonic vibration-assisted milling of aluminum alloy[J]. International Journal of Advanced Manufacturing Technology, 2012, 63(1-4): 41-49.

[19] CHERN G L, CHANG Y C. Using two-dimensional vibration cutting for micro-milling[J]. International Journal of Machine Tools \& Manufacture, 2006, 46(6): 659-666.

[20] RAZFAR M R, SARVI P, ZARCHI M M A. Experimental investigation of the surface roughness in ultrasonic-assisted milling $[\mathrm{J}]$. Proceedings of the Institution of Mechanical Engineers Part B Journal of
Engineering Manufacture, 2011， 225(9): 1615-1620.

[21］房善想, 赵慧玲, 张勤俭. 超声加工技术的应用现状及 其发展趋势[J]. 机械工程学报，2017，53(19)：22-32.

FANG Shanxiang, ZHAO Huiling, ZHANG Qinjian.The application status and development trends of ultrasonic machining technology[J]. Journal of Mechanical Engineering, 2017, 53(19): 22-32.

[22] 张翔宇, 隋翯, 张德远, 等. 高速超声振动切削钛合金 可行性研究[J]. 机械工程学报, 2017, 53(19): 120-127. ZHANG Xiangyu, SUI He, ZHANG Deyuan, et al. Feasibility study of high-speed ultrasonic vibration cutting titanium alloy[J]. Journal of Mechanical Engineering, 2017, 53(19): 120-127.

作者简介: 高泽, 男, 1993 年出生。主要研究方向为振动加工。 E-mail: sy1607403@buaa.edu.cn 张德远 (通信作者), 男, 1963 年出生, 博士, 教授, 博士研究生导师。 主要研究方向为难加工材料高效加工技术、振动切削与功率超声 技术。

E-mail: zhangdy@buaa.edu.cn 